Bull. Austral. Math. Soc.

VoL. 53 (1996) [135-142]

\title{
ABSTRACT DANIELL-LOOMIS SPACES
}

\author{
M. Díaz CarRillo and H. Günzler
}

In [3] for general integral metric $q$ an integral extension of Lebesgue power was discussed. In this paper we introduce the abstract Daniell-Loomis spaces $R_{p}, p$ real, $0<p<\infty$, of $q$-measurable functions with finite " $p$-norm", and study their basic properties.

\section{INTRODUCTION}

Recently in [3] an integral extension proceduce was given which works for general integral metric $q$. The basic ideas can be traced back to Loomis [9] and Schäfke [10]. One defines the extended functions of class $B^{q}$ of real-valued functions on a set $X$ with respect to a $B^{q}$ type seminorm. Using an appropriate local mean convergence we proved convergence theorems; and we introduced $q$-measurability, which is defined by the property that truncation by integrable functions leads to integrable functions. It allowed us to treat abstract Riemann, that is finitely additive, integration theory, as a fundamental example and applied simultaneously to Loomis's abstract Riemann integration, as well as to the Daniell and Bourbaki integration theories.

In this paper, using the method announced in [3] we shall give a presentation of the abstract Daniell-Loomis spaces $R_{p}, p$ real, $0<p<\infty$.

For nonnegative extended real-valued functions $f$ on $X$, if $p \geqslant 1, q_{p}(f)=$ $\left[q\left(f^{p}\right)\right]^{1 / p}$ satisfies the requirement of an integral metric, and essentially all the results discussed in [3] are true.

The relevant convergence properties with respect to $q$ or $q_{p}$ are developed. With weak continuity assumptions on the integral metric $q$, we prove as a fundamental result that the concepts of $q$ - and $q_{p}$-measurability are equivalent (Theorem 1 ).

This leads us to define the abstract Danniell-Loomis spaces $R_{p}$ as the class of $q$ measurable functions with finite $q_{p}(|\cdot|)$. The simple functions $B$ play the usual role in $R_{p}: R_{p}=B^{q_{p}}$ vector lattice (Theorem 2).

Finally, examples are presented which show that these results make it possible to study $\boldsymbol{R}_{\boldsymbol{p}}$-spaces for abstract Riemann or finitely additive, integration theory.

Received 4 April 1995

Copyright Clearance Centre, Inc. Serial-fee code: 0004-9729/96 \$A2.00+0.00. 


\section{NOTATION AND ASSUMPTIONS.}

In what follows we adhere to the notation and results of [3], and will be explained whenever necessary in order to make the paper self contained.

We extended the usual + to $\overline{\mathbb{R}} \times \overline{\mathbb{R}}$ by $r+s:=0$ if $r=-s \in\{\infty,-\infty\}$, $r-s:=r+(-s) . \overline{\mathbb{R}}_{+}:=[0, \infty], \overline{\mathbb{R}}:=\{-\infty\} \cup \mathbb{R} \cup\{\infty\}$.

We denote $a \vee b:=\max (a, b), a \wedge b:=\min (a, b)$ and $a \cap t:=(a \wedge t) \vee(-t)$ if $a, b \in \overline{\mathbb{R}}, t \in \overline{\mathbb{R}}_{+}$.

For an arbitrary nonempty set $X$ let $\overline{\mathbb{R}}^{X}$ consists of all functions $f: X \rightarrow \overline{\mathbb{R}}$. All operations and relations between functions are defined pointwise, with inf $\phi:=\infty$.

A functional $q: \overline{\mathbb{R}}_{+}^{X} \rightarrow \overline{\mathbb{R}}_{+}$is called an integral metric on $X$ if $q(0)=0$ and $q(f) \leqslant q(g)+q(k)$ if $f \leqslant g+k, f, g, k \in \mathbb{R}_{+}^{X}$.

If $B \subset \overline{\mathbb{R}}^{X}$, a function $f \in \overline{\mathbb{R}}^{X}$ is said to be $q$-integrable if it belongs to the closure of $B$ in $\overline{\mathbb{R}}^{X}$ with respect to $q$, that is there exists $\left(h_{n}\right) \subset B$ with $q\left(\left|f-h_{n}\right|\right) \rightarrow 0$ as $n \rightarrow \infty$.

$B^{q}$ denotes the set of all the $q$-integrable functions.

If additionally an $I: B \rightarrow \mathbb{R}$ is given which is uniformly continuous on $B$ with. respect to $q$, the unique $q$-continuous extension of $I$ to $B^{q}$ will be denoted $I^{q}$.

In all of the following, $B$ will be a function vector lattice in $\mathbb{R}^{X}$, that is a real linear space of functions under pointwise $=,+, \alpha$., such that $h \in B$ implies $|h| \in B$; then $k \wedge h, k \vee h \in B$ for $k, h \in B . I: B \rightarrow \mathbb{R}$ will be assumed linear with $I(h) \geqslant 0$ if $0 \leqslant h \in B$. Then, $q$-continuity of $I$ in 0 implies uniform $q$-continuity of $I$ on $B$.

We collect these assumption in

(1) $I, B$ as above, $q$ is an integral metric on $X$ and $I$ is $q$-continuous in 0 .

With (1), $B^{q}$ is closed with respect to $+, \alpha ., \vee, \wedge,|$.$| and I^{q}: B^{q} \rightarrow \overline{\mathbb{R}}$ is monotone, linear and $q$-continuous, (Theorem $1,[3]$ ).

A function $f \in \overline{\mathbb{R}}^{X}$ is said to be $q$-measurable if $f \cap h \in B^{q}$ for all $0 \leqslant h \in B$.

$M_{n}(q, B)$ denotes the set of all the $q$-measurable functions.

For convergence theorems we need a suitable local convergence in the mean of [3, p.414].

(2) For $f, f_{n} \in \overline{\mathbb{R}}^{X}, n \in \mathbb{N}, f_{n} \rightarrow f(q, B)$ means that for each $\varepsilon>0$ and $0 \leqslant h \in B$ there exists $n_{0}=n(\varepsilon, h) \in \mathbb{N}$ such that $q\left(\left|f-f_{n}\right| \wedge h\right)<\varepsilon$ if $n \geqslant n_{0},(q$-local convergence).

(3) Lebesgue's convergence theorem, (see Corollary VII, [3]): If (1) holds, $f_{n}, g \in B^{q}, f \in \overline{\mathbb{R}}^{X}$ is such that $f_{n} \rightarrow f(q, B)$ and $\left|f_{n}-f\right| \leqslant g, n \in \mathbb{N}$, then $f \in B^{q}$ and $q\left(\left|f_{n}-f\right|\right) \rightarrow 0$.

(4) For any integral metric $q$ and $M \subset \overline{\mathbb{R}}^{X}$ the corresponding local integral 
metric of Schäfke [10] (see also [3, p.416]) is defined by

$$
q_{\ell}(f):=\sup \{q(f \wedge h) ; 0 \leqslant h \in M\} \text { for all } f \in \overline{\mathbb{R}}_{+}^{X} .
$$

With (1), $q_{\ell}$ is again an integral metric such that $q_{\ell} \leqslant q$ and $q_{\ell}(f)=q(f)$ if $0 \leqslant f \leqslant g$ for some $g \in B^{q}$. One has $B \subset B^{q} \subset B^{q \ell}$ and $I^{q}=I^{q \ell}$ on $B^{q}$.

For further properties of $B^{q}$ and $B^{q \ell}$ see [3].

\section{2. $R_{p}$-SPACES}

For $q: \overline{\mathbb{R}}_{+}^{X} \rightarrow \overline{\mathbb{R}}_{+}, p$ real, $0<p<\infty$, with $f^{p}(t):=(f(t))^{p}, 0^{p}:=0, \infty^{p}:=\infty$, we define for all $f \in \overline{\mathbb{R}}_{+}^{X}$

$$
q_{p}(f):= \begin{cases}{\left[q\left(f^{p}\right)\right]^{1 / p}} & \text { if } p \geqslant 1 \\ q\left(f^{p}\right) & \text { if } 0<p<1 .\end{cases}
$$

Note that the case $p=1$ was studied in [3], and the natural question to consider is to what extent those results can be extended to values of $p$ other than 1 .

LEMma 1. (See Lemma 12, [3].) If $q:=\overline{\mathbb{R}}_{+}^{X} \rightarrow \overline{\mathbb{R}}_{+}$is an integral metric with $q(2 f)=2 q(f), 0<p<\infty$, then $q_{p}$ is also an integral metric on $X$, positivehomogeneous if $p \geqslant 1$.

Proof: Observe that $2 q(f) \leqslant q(2 f)$ implies $q(t f)=t q(f), 0<t<\infty$; also $|f+g|^{p} \leqslant f^{p}+g^{p}$ if $0<p \leqslant 1$.

If $p>1, q_{p}$ satisfies Minkowski's inequality for finitely-valued $f, g$, by Bourbaki $[2$, p.12].

Now, we denote $f_{e}(x):=f(x)$ if $f(x) \in \mathbb{R}, f_{e}(x):=0$ else, $f_{u}(x):=f(x)-f_{e}(x)$, $f_{\infty}:=f_{u} \vee 0$.

If $f, g \in \overline{\mathbb{R}}_{+}^{X}$ with $q_{p}(f), q_{p}(g)<\infty$, we have $\left[q_{p}(f+g)\right]^{p} \leqslant q\left[2^{p}\left(f^{p}+g^{p}\right)\right]<\infty$, and $\alpha q_{p}\left(f_{\infty}\right)=q_{p}\left(\alpha f_{\infty}\right) \leqslant q_{p}(f)<\infty$, so that $q_{p}\left(f_{\infty}\right)=0$.

Therefore $q_{p}(f+g) \leqslant\left[q_{p}(f+g)_{e}^{p}+0+0\right]^{1 / p} \leqslant q_{p}\left(f_{e}+g_{e}\right) \leqslant q_{p}\left(f_{e}\right)+q_{p}\left(g_{e}\right) \leqslant$ $q_{p}(f)+q_{p}(g)$.

For positive-homogeneous integral metric $q$, Hölder's inequality holds:

(6) Let $1<r, s<\infty$ be a pair of conjugate exponents, for functions $f, g \in$ $\overline{\mathbb{R}}_{+}^{R}$ then $q(f g) \leqslant q_{r}(f) q_{\bullet}(g)$.

(See for example [8, p.64-65], (6) follows with the aid of the expression $u v=$ $\inf \left\{(1 / p) t^{r} u^{r}+(1 / s) t^{-v} v^{2} ; t>0\right\}$ for real $u, v \geqslant 0$.)

For positive-homogeneous integral metrics $q$, Sections 1,2 of [3] hold for $B^{q_{p}}$ and

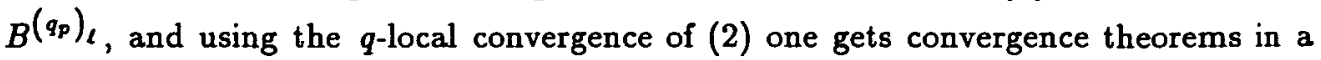
form analoguous to the classical ones. 
In order to obtain the full results one has to impose certain conditions upon $B$ and $q$.

(7) Let $q$ be a positive-homogeneous integral metric on $\overline{\mathbb{R}}_{+}^{R}$, and $0<p<\infty$. We assume

$|B|^{p}=|B|$ with $|B|:=\{h ; 0 \leqslant h \in B\}$.

$C_{0}(q, B): q(h \wedge t) \rightarrow 0$ if $0<t \rightarrow 0,0 \leqslant h \in B,(q$ continuous at 0$)$.

$C_{\infty}(q, B): q(h-h \wedge t) \rightarrow 0$ if $t \rightarrow \infty, 0 \leqslant h \in B,(q$ continuous at $\infty)$.

The above basic assumptions (1) and (7) will be retained in all that follows.

Observe that, with $|B|^{p}=|B|, C_{0}(q, B)$ implied $C_{0}\left(q_{p}, B\right)$.

Lеммa 2. Let $q$ be a positive-homogeneous integral metric, then $C_{\infty}\left(q_{p}, B\right)$ holds, that is, $q_{p}(h-h \wedge t) \rightarrow 0$ if $t \rightarrow 0,0 \leqslant h \in B$.

Proof: Case $1 \leqslant p<\infty$ : Observe that $a^{p}+b^{p} \leqslant(a+b)^{p}$ if $a, b \in \overline{\mathbb{R}}_{+}$. Thus, $(t-t \wedge s)^{p} \leqslant t^{p} \wedge s^{p}, t, s \in \overline{\mathbb{R}}_{+}$. Therefore $\left[q_{p}(h-h \wedge t)\right]^{p}:=q(h-h \wedge t)^{p}$ $\leqslant q\left(h^{p}-h^{p} \wedge s^{p}\right) \rightarrow 0$ if $s \rightarrow \infty$.

Case $0<p<1$ : We have $(h-h \wedge t)^{p}=((h-h \wedge t) / \varepsilon)^{p} \varepsilon^{p} \leqslant \varepsilon^{p}((h-h \wedge t) / \varepsilon)$ if $h \geqslant t+\varepsilon$ and $\leqslant \varepsilon \wedge h$ if $h<t+\varepsilon$. So that $(h-h \wedge t)^{p} \leqslant \varepsilon^{p-1}(h-h \wedge t)+\varepsilon \wedge h$.

Now, if $\varepsilon \rightarrow 0, \eta>0$, by $C_{0}(q, B), q(h \wedge \varepsilon)<\eta / 2$, and if $t \rightarrow \infty$, to $\eta>0$, by $C_{\infty}(q, B), \varepsilon^{p} q(h-h \wedge t)<\eta / 2$. Hence, one has $q_{p}(h-h \wedge t)=q\left[(h-h \wedge t)^{p}\right] \leqslant$ $q\left[\varepsilon^{p-1}(h-h \wedge t)\right]+q(h \wedge \epsilon)<\eta / 2+\eta / 2$, and the proof is complete.

The equivalence between $q$-convergence and $q_{p}$-convergence is made explicit in the following lemmas.

Lemma 3. Let $f, f_{n} \in \overline{\mathbb{R}}^{X}$, then $f_{n} \rightarrow f(q, B)$ implies $f_{n} \rightarrow f\left(q_{p}, B\right)$.

Proof: One can assume $f=0$ and $f_{n} \geqslant 0$. So, by (2) it suffices to show that given any $0 \leqslant h \in B$ if $q\left(f_{n} \wedge h\right) \rightarrow 0$ then $q_{p}\left(f_{n} \wedge h\right) \rightarrow 0$.

Case $1 \leqslant p<\infty$ : Choose $0 \leqslant h \in B, l_{n}:=f_{n} \wedge h$; by assumption $q\left(f_{n} \wedge h\right) \rightarrow 0$.

Now, if $0<t \in \mathbb{R},\left[q_{p}\left(l_{n}\right)\right]^{p}:=\left[\left(q\left(l_{n}^{p}\right)\right)^{1 / p}\right]^{p}=q\left(l_{n}^{p}\right)=q\left(f_{n}^{p} \wedge h^{p}\right) \leqslant q\left[f_{n}^{p} \wedge\right.$ $\left.\left(h^{p} \wedge t^{p}\right)\right]+q\left[\left(f_{n}^{p} \wedge h^{p}\right)-f_{n}^{p} \wedge\left(h^{p} \wedge t^{p}\right)\right] \leqslant q\left[f_{n}^{p} \wedge\left(h^{p} \wedge t^{p}\right)\right]+q\left(h^{p}-h^{p} \wedge t^{p}\right) \leqslant q\left(f_{n}^{p} \wedge\right.$ $\left.\left(h^{p} \wedge t^{p}\right)\right)+\varepsilon$, if $t>t_{\varepsilon, h}$, by $C_{\infty}(q, B)$.

One has, $l_{n}^{p} \wedge t^{p}=\left(l_{n} \wedge t\right)^{p}=t^{p}\left(\left(l_{n} \wedge t\right) / t\right)^{p} \leqslant t^{p}\left(\left(l_{n} \wedge t\right) / t\right)$, since $p \geqslant 1,0 \leqslant$ $\left(l_{n} \wedge t\right) / t \leqslant 1$.

Thus, if $t=t_{\varepsilon, h},\left[q_{p}\left(l_{n}\right)\right]^{p} \leqslant q\left(l_{n}^{p} \wedge t^{p}\right)+\varepsilon \leqslant q\left(t^{p}\left(\left(l_{n} \wedge t\right) / t\right)\right)+\varepsilon=\varepsilon+$ $t^{p}(1 / t) q\left(l_{n} \wedge t\right)=\varepsilon+t^{p-1} q\left(l_{n}\right)=\varepsilon+t^{p} q\left(f_{n} \wedge h\right) \leqslant 2 \varepsilon$, if $n \geqslant n_{\varepsilon}$.

Hence, $q_{p}\left(f_{n} \wedge h\right) \rightarrow 0$ as $n \rightarrow \infty$, for each $0 \leqslant h \in B$, that is $f_{n} \rightarrow 0\left(q_{p}, B\right)$. 
Case $0<p<1$ : We choose $0 \leqslant h \in B, t_{e, h}>0$ as above, and one has

$$
\begin{aligned}
q_{p}\left(f_{n} \wedge h\right):= & q\left[\left(f_{n} \wedge h\right)^{p}\right]=q\left(f_{n}^{p} \wedge h^{p}\right) \leqslant q\left(f_{n}^{p} \wedge h^{p} \wedge t\right) \\
& +q\left(h^{p}-h^{p} \wedge t\right) \leqslant q\left(f_{n}^{p} \wedge h^{p} \wedge t\right)+\varepsilon / 2
\end{aligned}
$$

if $t \geqslant t_{\varepsilon, h}$, by $C_{\infty}(q, B)$.

Hence, $q_{p}\left(f_{n} \wedge h\right) \leqslant q_{p}\left[\left(f_{n} \wedge s\right) \wedge h \wedge s\right]+\varepsilon / 2$, if $s=t^{1 / p} \geqslant t_{\varepsilon, h}$.

One can assume $f_{n} \leqslant s, h \leqslant s, s$ fixed, $s=t_{e, h}$.

If $A_{n, \delta}:=\left\{x \in X ; f_{n}(x) \geqslant \delta\right\}$, one gets $q_{p}\left(f_{n} \wedge h\right)=q\left(f_{n}^{p} \wedge h^{p}\right) \leqslant q\left[\left(s^{p} \chi_{A_{n, \delta}}\right) \wedge\right.$ $\left.h^{p}\right]+q\left(\delta^{p} \wedge h^{p}\right)$.

Since $0 \leqslant h^{p} \in B, C_{0}(q, B)$ gives $q\left(\delta^{p} \wedge h^{p}\right)<\varepsilon / 2$ if $\delta^{p} \leqslant \eta, 0<\eta<1$; hence, $q_{p}\left(f_{n} \wedge h\right) \leqslant s^{p} q\left(\chi_{A_{n, \delta}} \wedge\left(1 / s^{p}\right) h^{p}\right)+\varepsilon / 2$.

Furthermore, $\delta q\left(\chi_{A_{n, \delta}} \wedge\left(1 / s^{p}\right) h^{p}\right)=q\left(\delta \chi_{A_{n, \delta}} \wedge\left(\delta / s^{p}\right) h^{p}\right) \leqslant q\left[\delta \chi_{A_{n, \delta}} \wedge(h / s)^{p}\right]$ with $\delta$ fixed, $0<\delta<\min \left(1, \delta^{1 / p}\right)$.

Since $0 \leqslant(h / s)^{p}=(1 / s)^{p} h^{p} \in B$, there exists $n_{0}=n_{0}(\varepsilon, h, p, s, \delta)$ with $q\left[f_{n} \wedge\right.$ $\left.(h / s)^{p}\right]<\delta \varepsilon / 2 s^{-p}$ if $n \geqslant n_{0}$.

Hence, $q_{p}\left(f_{n} \wedge h\right) \leqslant \varepsilon / 2+\varepsilon / 2=\varepsilon$, hence $f_{n} \rightarrow 0\left(q_{p}, B\right)$. The proof is complete. $]$

We recall that in Lemma 3 , if $1 \leqslant p<\infty$ only $C_{\infty}\left(q,|B|^{p}\right)$ is needed. Also $q(k f)=k^{\delta} q(f)$ with $0<\delta<\infty, \delta$ fixed, independent of $f \in \overline{\mathbb{R}}_{+}^{X}$, instead of $q$ positive-homogeneous, is sufficient.

Lemma 4. Let $f, f_{n} \in \overline{\mathbb{R}}^{X}$, then $f_{n} \rightarrow f\left(q_{p}, B\right)$ implies $f_{n} \rightarrow f(q, B)$.

Proof: Case $1 \leqslant p<\infty$ : Use Lemma 3 for $1 / p \geqslant 1$, since $\left(q_{p}\right)_{1 / p}(f)=[q(f)]^{1 / p}$, then $q_{p}$ is again positive-homogeneous and the assumptions for $1 / p$ are fulfilled.

Case $0<p<1: q_{p}$ is not positive-homogeneous, one has only $q_{p}(s f)=s^{p} q_{p}(f)$, and the proof of the first part of Lemma 3 works also (with $1 / p$ instead of $p$ ), only in the last line one has, with $\bar{q}=q_{p}$ instead $q, t=t_{\varepsilon, h}$ fixed, $\bar{q}_{1 / p}\left(l_{n}\right)=q_{p}\left(l_{n}^{1 / p}\right)=$ $q\left(l_{n}\right) \leqslant \varepsilon+\bar{q}\left(t^{1 / p}\left(l_{n} \wedge t\right) / t\right)=\left(t^{1 / p-1}\right)^{p} \bar{q}\left(l_{n} \wedge t\right)+\varepsilon \leqslant \varepsilon+t^{1-1 / p} \bar{q}\left(l_{n}\right) \leqslant 2 \varepsilon$, if $n \geqslant n_{\varepsilon, t}$, or $q_{p}\left(l_{n}^{1 / p}\right)=\bar{q}\left(l_{n}\right) \leqslant 2 \varepsilon$, and thus the assertion holds.

Observe that $|B|^{p}=|B|$ implies $|B|^{1 / p}=|B|$, so, this condition is also true in Lemma 2 for $1 / p$.

The above results together with the Lebesgue convergence Theorem (3), is the key to proving that the concepts of $q$ - and $q_{p}$-measurability are equivalent.

THEOREM 1.

$$
M_{\cap}(q, B)=M_{\cap}\left(q_{p}, B\right)
$$


Proof: If $f \in M_{\cap}(q, B)$, by definition, for $0 \leqslant h \in B, f \cap h \in B^{q}$, so there are $h_{n} \in B$ with $h_{n} \rightarrow f \cap h(q, B)$; then also $h_{n} \cap h \rightarrow f \cap h(q, B)$. By Lemma 3, $h_{n} \cap h \rightarrow f \cap h\left(q_{p}, B\right)$. Since $\left|h \cap h-h_{n} \cap h\right| \leqslant 2 h$, the Lebesgue convergence theorem for $B^{q_{p}}$ (3), gives $f \cap h \in B^{q_{p}}$, for all $h \in B$, so that $f \in M_{\cap}\left(q_{p}, B\right)$.

On the other hand, if $f \in M_{\cap}\left(q_{p}, B\right)$, for all $0 \leqslant h \in B$ then $f \cap h \in B^{q_{p}}$, there are $h_{n} \in B$ with $h_{n} \rightarrow f \cap h\left(q_{p}, B\right)$. As above $h_{n} \cap h \rightarrow f \cap h(q, B)$, and the Lebesgue convergence theorem for $B^{q}$ yields $f \cap h \in B^{q}$, so $f \in M_{\cap}(q, B)$.

The class $R_{p}(B, I)$, or simply $R_{p}$, is defined as

$$
R_{p}(B, I):=\left\{f \in \overline{\mathbb{R}}^{X} ; f \text { is } q \text {-measurable and } q_{p}(|f|)<\infty\right\} .
$$

Our immediate goal is to show that, with additional weak assumptions on $q, R_{p}$ is a vector lattice aspace, and the "simple functions" $f \in B$ are dense in the metric $q_{p}(|\cdot|)$.

For this we need Definition 7 of $[3]$ and the following result concerning the $q_{\ell-}$ integrability of $q$-measurable functions $f$ with $q_{\ell}(|f|)<\infty$.

An integral metric $q$ is called $B$-semiadditive if one has

$$
0 \leqslant h_{n} \in B, \sup \left\{q\left(\sum_{i=1}^{n} h_{i}\right) ; n \in \mathbb{N}\right\}<\infty \Rightarrow q\left(h_{n}\right) \rightarrow 0 \text { as } n \rightarrow \infty,
$$

and $q$ is called $B$-additive if $0 \leqslant h, k \in B$ imply $q(h+k)=q(h)+q(k)$.

Obviously, $q B$-additive implies $q B$-semiadditive.

(8) If $q$ is $B$-semiadditive and $f$ is $q$-measurable such that $q_{\ell}(|f|)<\infty$, then $f \in B^{q \ell}[3$, Theorem 5].

We recall that by Lemma $1, q_{p}$ is an integral metric and $\left(q_{p}\right)_{\ell} \leqslant q_{p}$ on $\overline{\mathbb{R}}_{+}^{X}$.

ThEOREM 2. Let $q$ be $B$-semiadditive and $1 \leqslant p<\infty$ or $q B$-additive and $0<p<\infty$. Then $R_{p}:=\left\{f \in M_{\cap}(q, B) ; q_{p}(|f|)<\infty\right\}=B^{q_{p}}$.

Proof: By Theorem $1, f \in M_{\cap}(q, B)$ implies $f \in M_{\cap}\left(q_{p}, B\right)$, and if $q_{p}(|f|)<$ $\infty, q_{p} B$-semiadditive, by (8), $f \in B^{q_{p}}$.

Hence, it is enough to show that $q_{p}$ is $B$-semiadditive.

Case $1 \leqslant p<\infty$ : If $q$ is $B$-semiadditive, then $q\left(\sum_{1}^{n} h_{i}^{p}\right) \leqslant\left[q\left(\sum_{1}^{n} h_{i}\right)^{p}\right]=$ $q_{p}\left(\sum_{n}^{n} h_{i}\right)^{p}<k^{p}$ for all $n$. Hence, $q\left(h_{n}^{p}\right)=\left[q_{p}\left(h_{n}\right)\right]^{p} \rightarrow 0$, so that, $q_{p}\left(h_{n}\right) \rightarrow 0$, as
$n \rightarrow \infty$ 
Case $0<p<1: q$ is $B$-additive by assumption. Suppose that $q_{p}$ is not $B$ semiadditive, there exist $h_{n}$ with $q_{p}\left(h_{n}\right) \geqslant \varepsilon_{0}$ and $q_{p}\left(\sum_{1}^{m} h_{n}\right) \leqslant k$, for all $m \in$ $\mathbb{N}$. By Hölder's inequality, with $r=1 / p>1,1 / r+1 / s=1, m \varepsilon_{0} \leqslant \sum_{1}^{m} q_{p}\left(h_{n}\right)=$ $q\left(\sum_{1}^{m} h_{n}^{p} \cdot 1\right) \leqslant q\left[\left(\sum h_{n}^{p r}\right)^{1 / r} \cdot\left(\sum_{1}^{m} 1^{s}\right)^{1 / s}\right]=q_{p}\left(\sum_{1}^{m} h_{n}\right) m^{1 / s} \leqslant k m^{1 / r}$ or $m^{1-1 / s} \leqslant$ $k / \varepsilon_{0}$ a contradiction.

Finally, observe that if $|B|^{p}=|B|, f \in B^{q_{p}}$ implies $q_{p}(|f|)<\infty$. One has the above equality if $q(h)<\infty$ for each $0 \leqslant h \in B$, and the proof is completed.

Note that $q$-semiadditive is not needed in Theorem 1 .

Let $N_{p}=N_{p}(B, I):=\left\{f \in \overline{\mathbb{R}}^{X} ; q_{p}(|f|)=0\right\}$ ( $q$-nulfunctions).

One has $B \cup N_{p} \subset R_{p}, N_{p}$ is closed with respect to,,$+- \alpha .,|$.$| .$

For all $f, g \in \overline{\mathbb{R}}^{X}, f=g\left(q_{p}\right)$ means that $f-g \in N_{p}$, (see [3, p.412-413]).

Since $q_{p}(|f-g|)=0$ implies $f=g\left(q_{p}\right)$, strictly speaking, the elements of $R_{p}$ are equivalence class of functions defined on $X$.

With Theorem 2 the theory of integration presented in [3] is available.

\section{Applications and examples (See Section 3 of [3].)}

1. With $q(f)=I^{-}(f):=\inf \{I(g) ; f \leqslant g \in B\}$ for all $f \in \overline{\mathbb{R}}_{+}^{X}$, one has $B^{q}=$ $R_{\text {prop }}(B, I)$ (proper Riemann- $I$-integrable functions or the "two-sided completion" of Loomis $[9, p .170])$.

If $q_{\ell}(f)=I_{\ell}^{-}(f)$ (of Definition (4)), one gets $R_{1}(B, I):=B^{q}=$ closure of $B$ in $\overline{\mathbb{R}}^{X}$ with respect to the distance $d(f, g):=\left(I_{\ell}^{-}\right)(|f-g|$ ) (abstract Riemann- $I$-integrable functions of [4]), containing the "one-sided completion" of Loomis [9, p.178]).

$I^{-}$and $I_{\ell}^{-}$are positive-homogeneous integral metrics on $\overline{\mathbb{R}}_{+}^{X}$, also they are $B$ additive. Here, $R_{p}(B, I)=B^{\left(I_{\ell}^{-}\right) p}$.

We recall that $I_{\ell}^{-}$is the "essential upper functional" associated with $I^{-}$in the sense of Agner and Portenier [1], so that, $R_{1}(B, I)$ is the set of all the essentially integrable functions (with respect to $I^{-}$). Also, in Gould [6], Stone's axiom $B \wedge 1 \subset B$ is assumed, so by [7] his results are already subsumed by the $R_{1}$-space.

2. We consider now $B, I$ arising from finitely-additive set functions $\mu$, with arbitrary set $X$.

$\Omega$ is a semiring of sets from $X, \mu: \Omega \rightarrow \mathbb{R}_{+}$is finitely additive on $\Omega, B=B_{\Omega}=$ real-valued step functions on $\Omega$, and $I=I_{\mu}=\int d_{\mu}$ on $B_{\Omega}$.

With $q=I_{\mu}^{-}, q_{\ell}=\left(I_{\mu}^{-}\right)_{\ell}$ one has $B_{\Omega}^{q}=R_{\text {prop }}(\mu, \Omega)$ (abstract proper Riemann- 
$\mu$-integrable functions) and $B_{\Omega}^{q \ell}=R_{1}(\mu, \Omega)$ (Riemann- $\mu$-integrable functions of [7]), which contains $L(X, \Omega, \mu, \mathbb{R})$ of Dunford-Schwartz [5].

In this situation, $I_{\mu}^{-}$is $B_{\Omega}$-additive and a positive-homogeneous integral metric on $X$. Also, $B_{\Omega}$ is Stonian, $C_{\infty}\left(I_{\mu}^{-}, B_{\Omega}\right)$ and $C_{0}\left(I_{\mu}^{-}, B_{\Omega}\right)$ of $(7)$ hold.

With (1), if $I$ satisfies Daniell's condition (or $I$ is $\sigma$-continuous), that is, $I\left(h_{n}\right) \rightarrow 0$ whenever $0 \leqslant h_{n} \in B, h_{n} \geqslant h_{n+1} \rightarrow 0$ pointwise on $X$, one has that $q=I^{\sigma}(f):=$ $\inf \left\{\sum_{n=1}^{\infty} I\left(h_{n}\right) ; h_{n} \in B, f \leqslant \sum_{n=1}^{\infty} h_{n}\right\}$ for all $f \in \overline{\mathbb{R}}_{+}^{X}$, is the induced $B$-additive integral norm with Daniell's $L^{1}=B^{q}$.

Finally, if $\Omega$ is a $\sigma$-ring and $\mu$ is $\sigma$-additive, then $R_{q}(\mu, \Omega)=L^{1}(\mu, \Omega)$ modulo nulfunctions by $[7, \mathrm{p} .265]$.

\section{REFERENCES}

[1] B. Anger and C. Portenier, Randon integral (Birkhäuser, Basel, 1992).

[2] N. Bourbaki, Intégration. Elements de Mathematique XIII, Livre VI (Hermann, Paris, 1952).

[3] M. Díaz Carrillo and H. Günzler, 'Local integral metrics and Daniell-Loomis integrals', Bull. Austral. Math. Soc. 48 (1993), 411-426.

[4] M. Díaz Carrillo and P. Muñoz Rivas, 'Positive linear functionals and improper integration', Ann. Sci. Math. Québec 18 (1994), 149-157.

[5] N. Dunford and J.T. Schwartz, Linear operators I (Interscience, New York, 1957).

[6] G.G. Gould, 'The Daniell-Bourbaki integral for finitely additive measures', Proc. London Math. Soc. 16 (1966), 297-230.

[7] H. Günzler, Integration (Bibliogr. Institut, Mannheim, 1985).

[8] H. König, 'Daniell-Stone integration without the lattice condition and its application to uniform algebras', Ann. Univ. Sarav. Ser. Math. 4 (1992).

[9] L.H. Loomis, 'Linear functionals and content', Amer. J. Math. 76 (1956), 168-182.

[10] F.W. Schäfke, 'Integrationstheorie I', J. Reine Angew. Math. 244 (1970), 154-176.

Departamento de Análisis Matemático

Universidad de Granada

Granada 18071

Spain
Mathematisches Seminar

Universitát Kiel

D 24098 Kiel

Germany 